\title{
Transport Stream Files Assembling and Analysis of BER Performance into ISDB-Tb Standard
}

\author{
A. Raizer \\ K. Toccolini \\ M. P. Fonseca
}




\title{
Transport Stream Files Assembling and Analysis of BER Performance into ISDB-Tb Standard
}

\author{
A. Raiz , K. Toccolini and M. P. Fonseca
}

\begin{abstract}
This work presents a technique for assembling a TS (Transport Stream) file, in accordance with the ISDB-Tb (Integrated Services Digital Broadcasting Terrestrial Brazilian) standard, in order to transmit it through a digital TV signal generator to analyze the parameters variation effects of the ISDB-Tb system in the BER (Bit Error Rate) performance. For this analysis, measurements were taken in three different communication channels, with distinct features. In each channel, the 16QAM and 64QAM modulations were evaluated, and several combinations were tested among the following parameters: operating mode, code rate, guard interval and time interleaving. Also, BER measurements were taken for a $\mathrm{C} / \mathrm{N}$ (Carrier to Noise) range, set from transmitter, then the $E_{b} / N_{o}$ ratio was calculated, which allowed to plot curves for each measure.
\end{abstract}

Index Terms-Bit Error Rate, Communication Channels, Digital TV, ISDB-Tb, Transport Stream.

\section{INTRODUCTION}

$\mathrm{I}_{\mathrm{T}}^{\mathrm{n}}$ $\mathrm{n}$ recent years, Brazil has developed and adopted the ISDB$\mathrm{Tb}$ (Integrated Services Digital Broadcasting Terrestrial Brazilian) standard for digital TV transmission. A transition from analog to digital transmission is being conducted slowly, although some Brazilian cities already are using only digital signal. Since this system became a standard for TV broadcasters in Brazil, techniques have been popularized to create files to be transmitted, also, it has become required a study of how each standard parameter changes the transmission system robustness.

The ISDB-Tb standard determines broadcasting characteristics, such as the use of OFDM (Orthogonal Frequency Division Modulation) for its transmission. One of the main advantages of OFDM is that its transmission is based on band segmentation, which allows flexibility of operation by segment as well as greater robustness regarding the frequency selectivity of the communication channel [1].

The broadcasting of the different segments originates the hierarchical transmission term. Segments are composed of

This paper was supported financially by the company W3Sat through the research project titled "Development of antennas for reception of digital signals on high definition television (HDTV)".

A. Raizer (adroaldo.raizer@ufsc.br), K. Toccolini (kassia.t@posgrad.ufsc.br) and M. P. Fonseca (gerencia.maglab@contato.ufsc.br) are within GEMCO - Engineering and Electromagnetic Compatibility Group; MagLab - Electromagnetism and Electromagnetic Compatibility Laboratory; Federal University of Santa Catarina (UFSC); P.O.Box 5024; Florianópolis-SC, Brazil, ZIP Code 88040970 . subcarriers, since they are independent, they can send different data in the same channel. ISDB-Tb standard imposes a $6 \mathrm{MHz}$ band for each channel, with 14 segments, and one of them is used as a security interval in the channel edges. Therefore, there remain 13 segments to be distributed in up to three independent layers (A, B and C) [2].

The ISDB-Tb system also allows setting several transmission parameters in the transmitter. Each parameter can be defined in order to fit the transmission application and the desired robustness of the system [2]. Between the parameters that can be defined in the transmission, the following ones were analyzed in this paper: number of segments, modulation type, hierarchical processing, code rate, time interleaving, operating mode and guard interval. The last two modify all the segments of the channel, while the others can be selected for each layer of the hierarchical transmission.

In addition to ISDB-Tb standard defines broadcasting features, it specifies rules for TS (Transport Stream) files that are transmitted. Since devices that are able to assemble TS files are expensive Software implementation has become a low cost alternative which also allows laboratory testing beforehand. As proposed in [3], it is possible through the free software FFMPEG [4] coding files, which can be used for this purpose. In this work, it was proposed a way with few configurations of assembling TS to transmit the desired video to a TV.

As proposed in [5] and [6], one of the ways to evaluate the performance of the modulation is to plot the BER (Bit Error Rate) by $E_{b} / N_{o}$ curve. This work proposes to perform BER (Bit Error Rate) measurements by varying the $\mathrm{E}_{\mathrm{b}} / \mathrm{N}_{\mathrm{o}}$ through a digital TV signal transmitter, in order to verify the relation of the parameters that are flexible in the ISDB-Tb standard with the BER measured by the receiver.

\section{Methodology}

To carry out the tests of different channel settings, it was necessary to assemble a TS file as the input of the digital TV signal transmission equipment. With the input file, three different communication channels were tested. The possible parameters of the system were varied and the bit error rate were measured.

The bit error rate (BER) is an important measure to characterize the statistical performance of a digital communication system, and it is defined as the ratio of the error number of bits detected to the total number of bits received [7]. 


\section{A. Assembling of TS}

The assembling of TS was done through the free software FFMPEG [4], which allows an H.264 video encoding and AAC audio encoding, requisites for ISDB-Tb standard [3]. In addition, a multiplexing of the audio and video signals was done by setting up a TS file in the MPEG-2 standard. The MPEG-2 standard defines how audio, video and data components can be combined into a single file, which is called multiplexing [8]. The multiplexing, besides being done with audio, video and data components can be used to multiplex more than one TS to take advantage of the hierarchical transmission, and to send one or more programs at the same time. In this work, only one TS file was used in the final transmission because of the characteristics of the transmitter that was used, which implies in the use of just one layer.

In addition to the audio and video encodings, an important definition in this step is the output bit rate of the multiplexing, since it is the value which will be the bit transfer rate. Hence, as the variation of channel parameters influences the available band, it influences the possible bit rate to be transmitted.

Therefore, a TS file with HD resolution was assembled (1280x720), with a bit rate of $4 \mathrm{Mbps}$. This rate allows the use of this file in different configurations. Fig. 1 shows how FFMPEG was used to assemble the described file. In addition to the parameters mentioned, other settings have been performed to fit to in the ISDB-Tb standard. Also, it was changed the channel name information, in this case, GEMCO, and, the output file was named HD.ts.

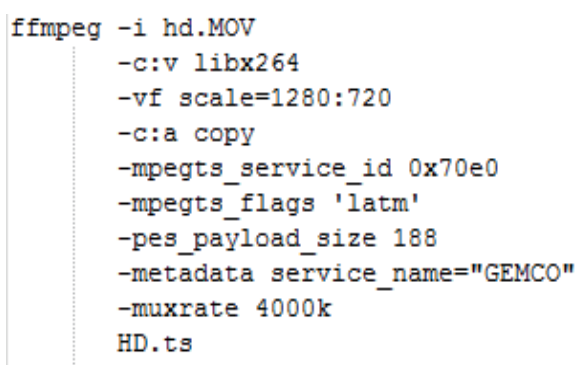

Fig. 1. Assembling of TS. Source code used to assemble the TS file to be transmitted as digital TV signal.

\section{B. Transmission Frequency}

In order to transmit the digital TV signal, the SFE100 (Rohde \& Schwarz) was used as a transmission signal generator for testing systems. In this transmitter, it is necessary to determine the frequency at which the signal will be transmitted. In this case, it was verified a frequency that was not occupied to carry out the tests, and it was chosen the value of $491.143 \mathrm{MHz}$.

From this definition, the assembled TS file was transmitted to a digital TV device in order to validate it. In the Fig. 2, it is possible to observe that the video is being transmitted to channel 17, which matches with the $491.143 \mathrm{MHz}$ frequency. Another data, visible in the Fig. 2, is the name of the channel, given from coding as GEMCO.

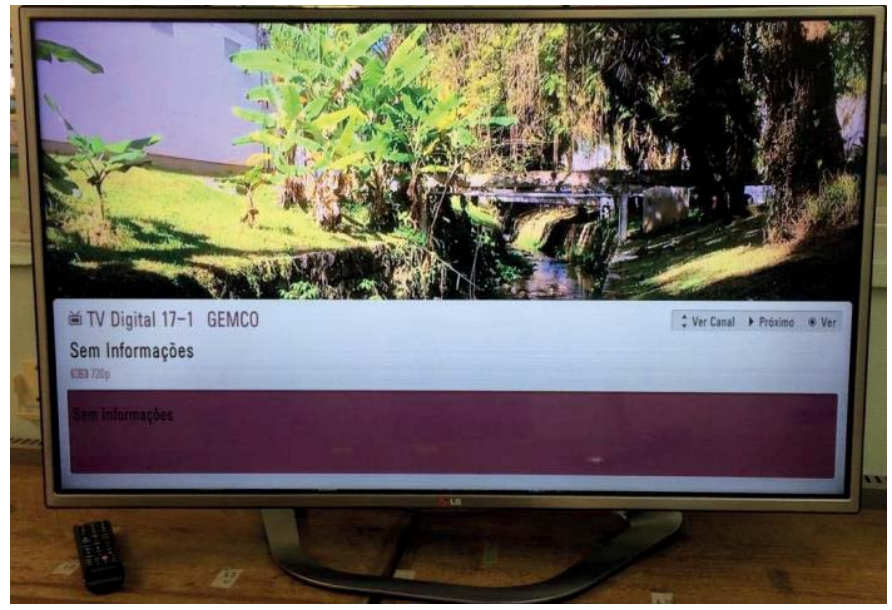

Fig. 2. TS file validation. Transmission of TS assembled to a TV to verify the correct file encoding.

\section{C/N Variation}

SFE100 allows transmitting the signal desired along with controlled AWGN (Additive White Gaussian Noise) noise. This control can be done through the $\mathrm{C} / \mathrm{N}$ (Carrier to Noise) ratio in $\mathrm{dB}$ (decibel). The $\mathrm{C} / \mathrm{N}$ variation values were different for each channel, since this affects the decoding and BER measurement done by the receptor.

\section{Channels Under Tests}

BER measurements were performed in three different communication channels. The first channel tested was the ideal channel. To simulate this idealization the transmitter was connected directly to the receiver through a cable, in this way, the errors detected by the receiver would be only from the noise generated by the transmitter.

For the second channel tested, the receiver was kept near the transmitter, to avoid reflection, and to simulate an environment where there was no multipath.

Finally, the third channel tested was with the receiver away from the transmitter, with several metallic obstacles along to simulate a multipath environment.

\section{E. The Varied Parameters}

To use the SFE100, the TS input file has to be transmitted in just one layer. Thereby, it was chosen to perform the tests with layer $\mathrm{C}$, with 9 segments of the hierarchical transmission of the ISDB-Tb standard. In addition, the parameters of operation mode, guard interval, code rate, time interleaving, and modulation type were varied. For the measurements, the minimum and maximum values of each parameter were used. Table 1 shows the parameters tested in channels 1 and 2, totaling 32 measurements for these two channels. Also, Table 2 shows the parameters tested in channel 3, which were tested for the necessary variations for further comparison, totaling 16 measurements. 
Table 1 - Varied Parameters in Channels 1 and 2.

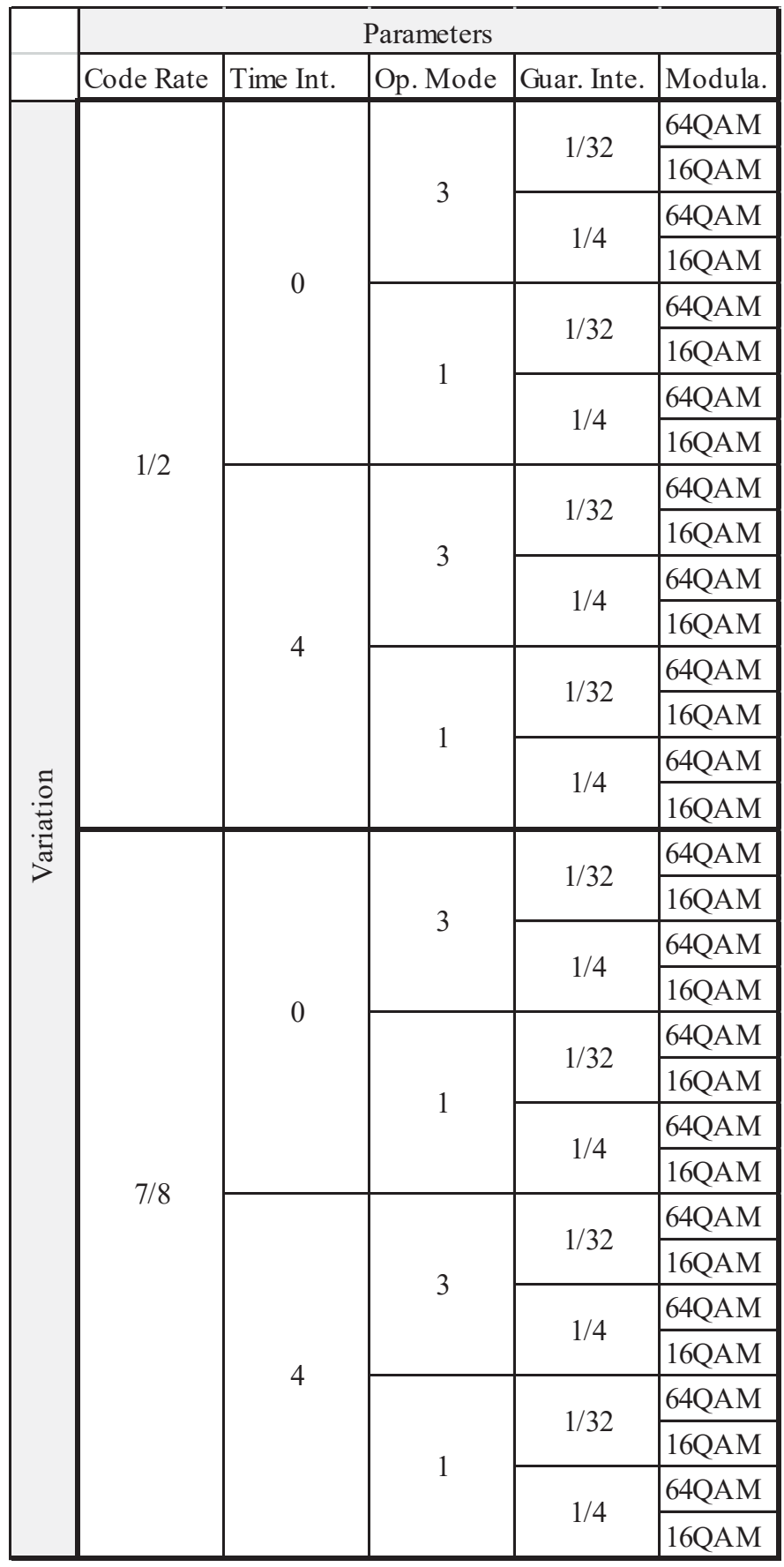

\section{F. BER Measurement}

To measure the BER of each tested digital TV system configuration, on each channel, a digital TV signal analyzer equipment - ETH Handheld TV Analyzer, Rohde \& Schwarz - was used. This equipment decodes the received digital TV signal, taking measurements of BER and MER, and allowing to verify the received settings. Particularly in the BER measurements, ETH emulates two error correction codes: Viterbi and Reed Solomon [9] [10], used in digital TV receivers, and the bit error rate can be evaluated before and after each of these codes. As this work intends to analyze how the variations of the parameters of the ISDB-Tb system
Table 2 - Varied Parameters in Channel 3

\begin{tabular}{|c|c|c|c|c|c|}
\hline & \multicolumn{5}{|c|}{ Parameters } \\
\hline & Code Rate & Time Int. & Op. Mode & Guar. Inte. & Modula. \\
\hline \multirow{16}{*}{ 皃 } & \multirow{16}{*}{$1 / 2$} & \multirow{8}{*}{0} & \multirow{4}{*}{3} & \multirow{2}{*}{$1 / 32$} & 64QAM \\
\hline & & & & & 16QAM \\
\hline & & & & \multirow{2}{*}{$1 / 4$} & 64QAM \\
\hline & & & & & 16QAM \\
\hline & & & \multirow{4}{*}{1} & \multirow{2}{*}{$1 / 32$} & 64QAM \\
\hline & & & & & 16QAM \\
\hline & & & & \multirow{2}{*}{$1 / 4$} & 64QAM \\
\hline & & & & & 16QAM \\
\hline & & \multirow{8}{*}{4} & \multirow{4}{*}{3} & \multirow{2}{*}{$1 / 32$} & 64QAM \\
\hline & & & & & 16QAM \\
\hline & & & & \multirow{2}{*}{$1 / 4$} & 64QAM \\
\hline & & & & & 16QAM \\
\hline & & & \multirow{4}{*}{3} & \multirow{2}{*}{$1 / 32$} & 64QAM \\
\hline & & & & & 16QAM \\
\hline & & & & \multirow{2}{*}{$1 / 4$} & 64QAM \\
\hline & & & & & 16QAM \\
\hline
\end{tabular}

influence the bit error rate, it was observed the measure before error correction codes.

\section{G. Analysis of the BER to $E_{b} / N_{o}$ Relation}

An important way to analyze the BER is its relation to the $\mathrm{E}_{\mathrm{b}} / \mathrm{N}_{\mathrm{o}}$ ratio, and this ratio is defined as the SNR (Signal Noise Ratio) normalized per bit, without taking the bandwidth into consideration.

Since SFE100 allows the variation of $\mathrm{C} / \mathrm{N}$, the equation presented in (1) was used to obtain the $\mathrm{E}_{\mathrm{b}} / \mathrm{N}_{\mathrm{o}}$ [11].

$$
\frac{E_{b}}{N_{o}}=\frac{C}{N} * \frac{B}{f_{b}}
$$

With:

$$
\begin{aligned}
& \mathrm{B}=\text { Channel Bandwidth } \\
& \mathrm{f}_{\mathrm{b}}=\text { Net Bitrate }
\end{aligned}
$$

In line with that, it was used an $\mathrm{f}_{\mathrm{b}}$ of $4.01 \mathrm{Mbps}$, that was the bit rate set in the TS assembled file. In addition, the bandwidth was used which belonged only to the $\mathrm{C}$ layer, that is, the bandwidth that corresponds to 9 segments, which is 3.86MHz.

After the measurements were taken, the data was compiled and the curves were plotted in MATLAB ${ }^{\circledR}$ software [12].

\section{RESULTS}

The results will be presented first referring to software assembled TS, followed by the BER curves plotted for each communication channel tested (1,2 and 3) with 16QAM and 64QAM modulations. 


\section{A. TS File Assembled}

The ffprobe tool, of FFMPEG, allows taking information about multimedia streams. In the Fig. 3, this tool was used to show the details about the source file, used to created TS final file. As can be seen, several features are presented, including audio and video coding, highlighting its high resolution and bitrate, which make impossible to multiplex this file directly in a TS.

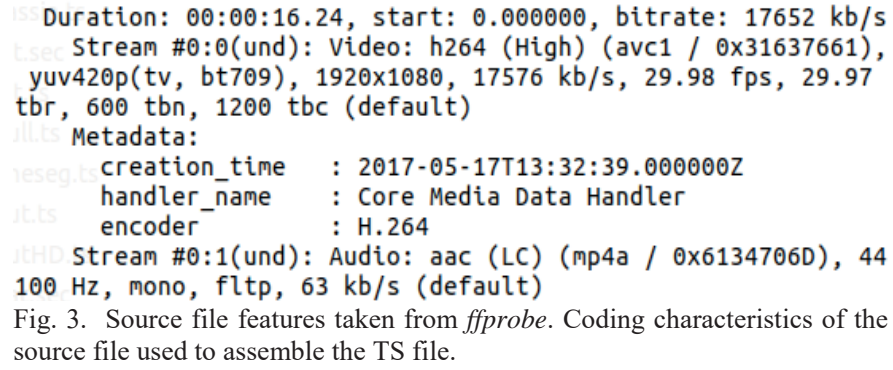

Fig. 3. Source file features taken from ffprobe. Coding characteristics of the source file used to assemble the TS file.

Using the same tool (ffprobe), Fig. 4 shows the file encoded in audio and video, and multiplexed in the MPEG-2 standard, as required. In this file, it is possible to observe an HD resolution set, bitrate at $4.01 \mathrm{Mbps}$, also it has a channel name information.

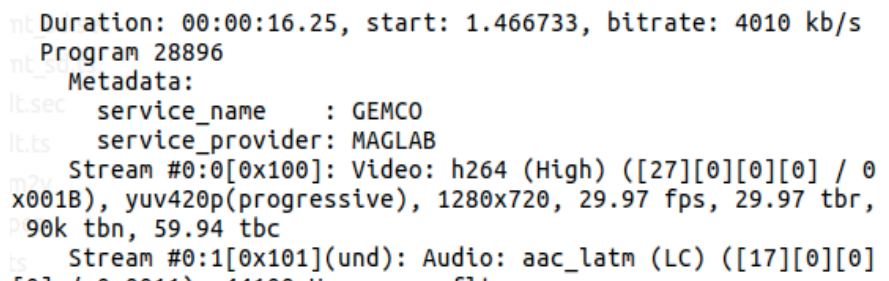

Fig. 4. TS Assembled File. File encoded in conform to ISDB-Tb standard, HD resolution, multiplexed, ready to be transmitted.

\section{B. Comparison of Different Parameters in the Channels Tested}

All the curves obtained in channel 1 with 16QAM modulation were plotted; the results are shown in Fig. 5, where can be noticed that the variation of BER is only perceived when varied between modes of operation 1 and 3 . Other variations originated values close to and even equal to each other, which indicates that the variation of the others ISDB-Tb system parameters do not change the bit error rate.

In these measurements, the greatest change observed in the bit error rate is when it is varied between operating modes 1 and 3. Even as 16QAM modulation, in general, other parameters do not change bit error rate performance as much. However, in this case, one curve did not follow the same pattern, which has the guard interval of $1 / 32$, code rate of $1 / 2$ and time interleaving of 0 , belongs to mode of operation 3 . This means this parameters combination is the one that has the best BER performance between the operating mode 3 , since the other ones need more power to have the same bit error rate.

Comparing Fig. 5 and Fig 6, it can be seen that 16QAM modulation has a more robust configuration, requiring less energy to have the same BER as 64QAM.

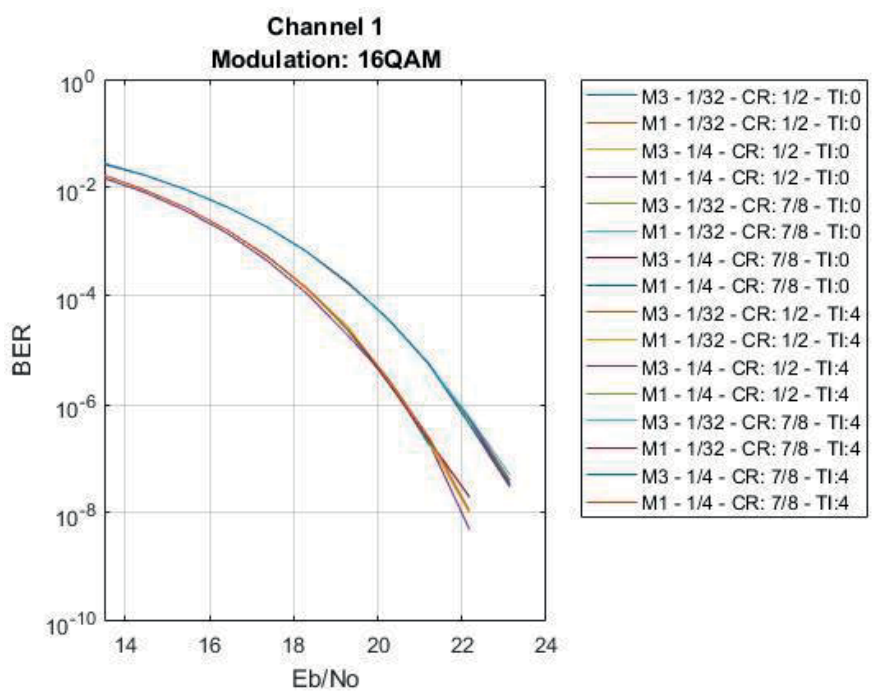

Fig. 5. Channel 1. Modulation 16QAM. Plotted curves for 16QAM modulation of the measurements performed on channel 1 .

For the 64QAM modulation, the curves of channel 1 were plotted as and are shown in Fig. 6.

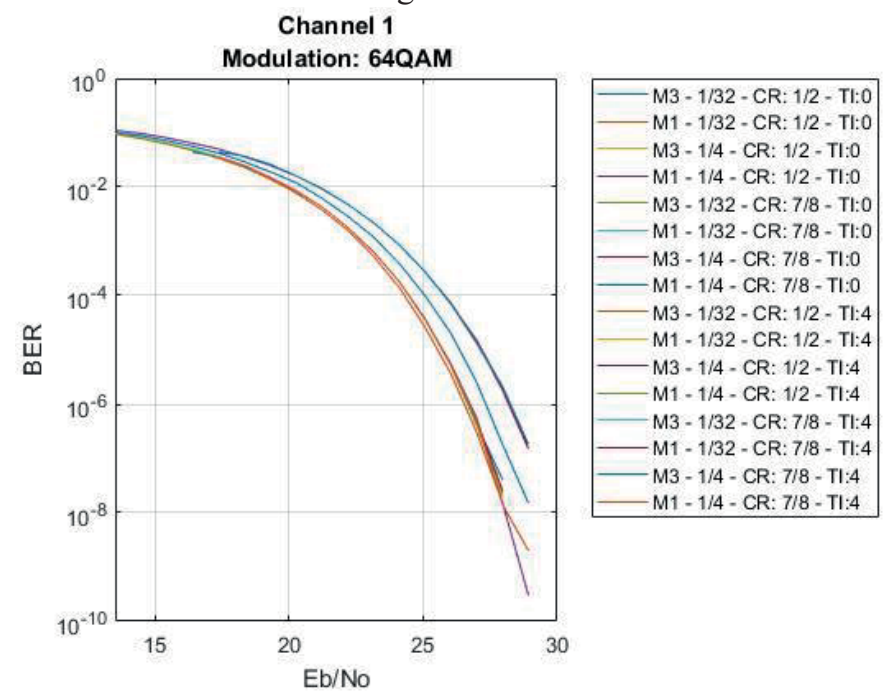

Fig. 6. Channel 1. Modulation 64QAM. Plotted curves for 64QAM modulation of the measurements performed on channel 1 .

In the same way as the previous one, curves were plotted for channel 2, which is the measurement with the transmitting and receiving antennas close and with direct view. Fig. 7 shows the measurements taken for this channel with 16QAM modulation.

As in channel 1, it is noticed, in this case, the changes in parameters, except operating mode, do not affect the BER. In comparison with channel 1 , with the same modulation, it is noticed that the BER remained almost with the same values. 


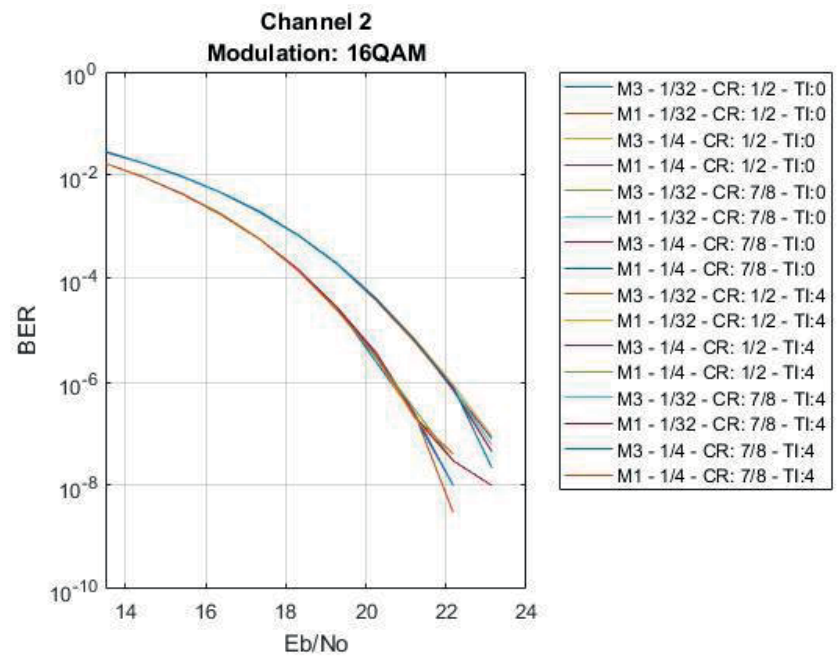

Fig. 7. Channel 2. Modulation 16QAM. Plotted curves for 16QAM modulation of the measurements performed on channel 2 .

Also for channel 2, the curves for 64QAM modulation, were plotted and are shown in Fig. 8.

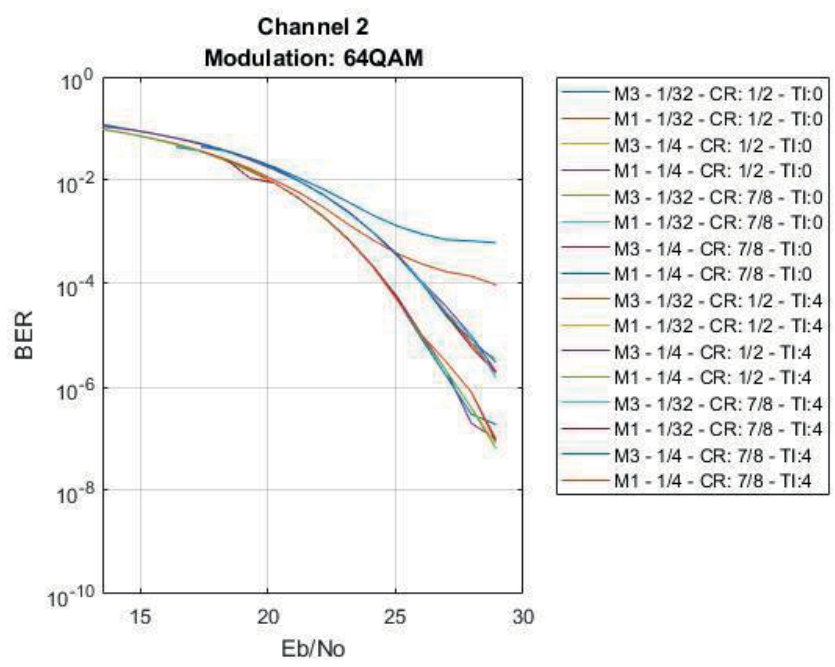

Fig. 8. Channel 2. Modulation 64QAM. Plotted curves for 64QAM modulation of the measurements performed on channel 2 .

In this case, it can be observed the previously verified pattern. The most effective way of improving the BER is by changing the operating mode, since other parameters did not change it significantly. However, there are two curves that do not follow the same characteristics as the other ones. These curves have the same combination of curve parameters highlighted in channel 1 (guard interval of $1 / 32$, code rate of $1 / 2$ and time interleaving of 0 ), but now for mode 1 as well. On the other hand, in this channel this combination proved to be less efficient than the others in BER performance.

As in channel 1, higher power is required with a 64QAM modulation to have the same 16QAM bit error rate.

When measurements were taken for channel 3, it was possible to measure a lower $\mathrm{C} / \mathrm{N}$ value when compared to the other two channels. This occurred since at a particular threshold value (depending on parameters) the AWGN noise added by the transmitter generated a BER that was equal to the existing BER in the communication channel, which made it impossible to decrease the BER by increasing only the $\mathrm{C} / \mathrm{N}$ of the transmitter. The alternative to increase the power transmission was not used to maintain the standard used in the other two channels measurements.

The measurements performed for channel 3, with 16QAM and 64QAM modulations, were plotted and are shown in Fig. 9 and Fig. 10, respectively.

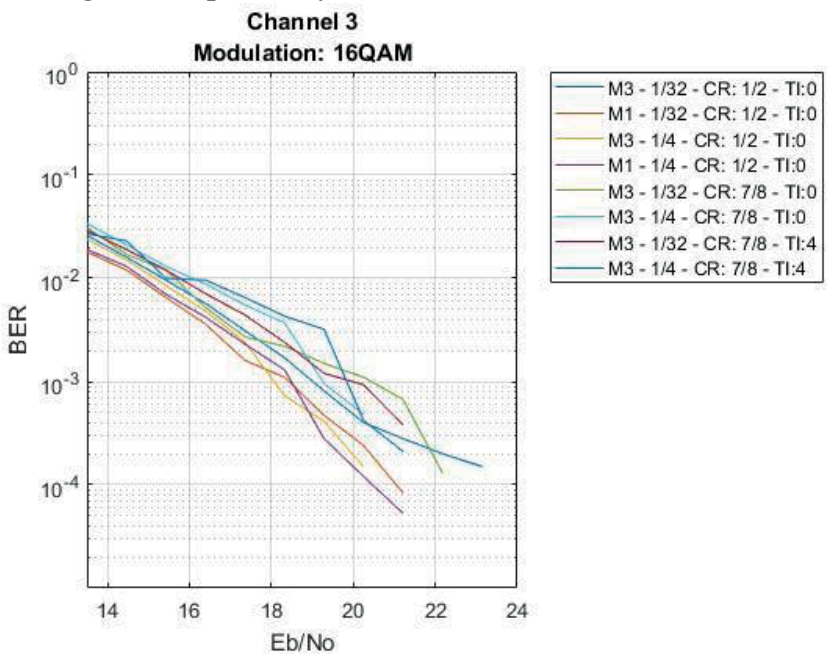

Fig. 9. Channel 3. Modulation 16QAM. Plotted curves for 16QAM modulation of the measurements performed on channel 3 .

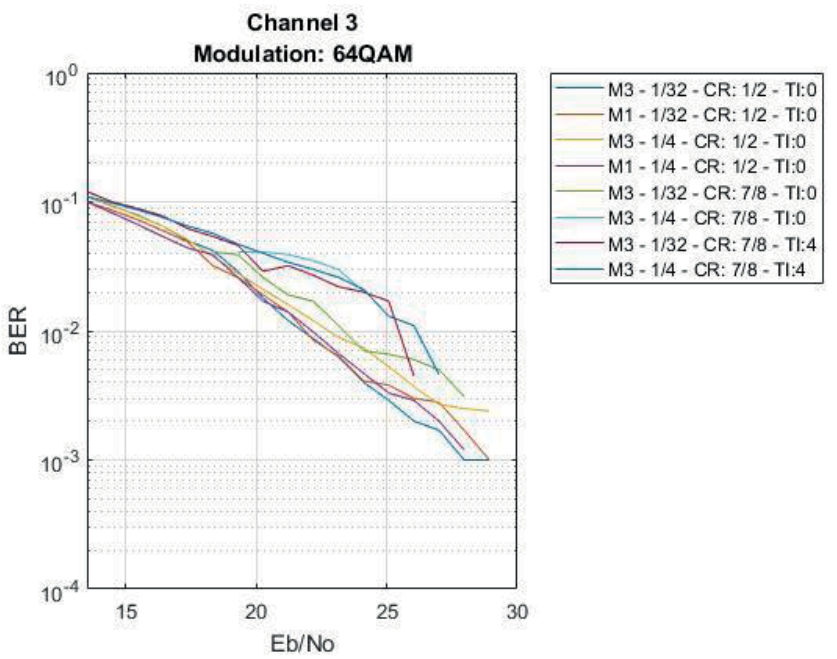

Fig. 10. Channel 3. Modulation 64QAM. Plotted curves for 64QAM modulation of the measurements performed on channel 3 .

In channel 3, it cannot be perceived a curve pattern, like channels 1 and 2 . In addition, while in a BER of $10^{-3}$ in channels 1 and 2, the $\mathrm{E}_{\mathrm{b}} / \mathrm{N}_{\mathrm{o}}$ value varied from $17 \mathrm{~dB}$ to $18 \mathrm{DB}$ at 16 QAM, in channel 3 the $E_{b} / N_{o}$ value for this BER value ranged from $18 \mathrm{~dB}$ to $21 \mathrm{~dB}$.

This comparison shows that in channel 3 there is more AWGN noise that degrades the signal, and there are noises from different natures. The presence of other types of noise, which changes the pattern of previously obtained curves, leads to the conclusion that there is no direct relationship between the improvement of BER and the change of some specific ISDB-Tb system parameter. In channel 3, which is equivalent to a real case where there are losses and noise of different natures, no evidence was found of a combination that provides 
a better performance of BER. If it is desired to improve the BER in a certain transmission, it would be better to take measurements for each communication channel specifically, in order to find the best combination that provides this efficacy.

\section{CONCLUSION}

In this paper, BER measurements were presented for different combinations of ISDB-Tb transmission system configurations. In addition, to achieve these measures, a TS file was assembled for transmission using only the FFMPEG software.

With the implementation of the ISDB-Tb standard, the FFMPEG software tool proved effective for the assembling of TS test files for laboratory measurements. The TS file could be transmitted to a TV; also it was correctly decoded and analyzed by a digital TV signal analyzer.

With the measurements of BER $x \mathrm{E}_{\mathrm{b}} / \mathrm{N}_{\mathrm{o}}$ in this paper, it can be seen that if there is a high bit error rate problem in the digital TV transmission, changes in the parameters of the ISDB-Tb system may or may not improve the performance. Since in a controlled environment where there was only AWGN noise, a pattern could be observed, whereby using operation mode 1 (where a smaller number of subcarriers are used) the performance of the BER improves. However, in the case of channel 3, where there was signal degradation for reasons other than the AWGN, the behavior of the curves did not follow a pattern. With this, it is concluded that in real transmission environments, which resemble channel 3 , to find the best combination for a good performance of BER, new measurements and tests should be carried out for the specific communication channel.

\section{REFERENCES}

[1] D. Villamarín, M. A. Illescas, G. Olmedo and R. L. Cueva, "Generating a transport stream for digital terrestrial television system in conformance with ISDB-Tb standard," presented at IEEE Colombian Conference on Communications and Computing (COLCOM), Medellin, Colombia, May 22-24, 2013.

[2] FFMPEG software. [Online]. Available on https://ffmpeg.org/download.html.

[3] Digital terrestrial television - Transmission system, ABNT NBR 15601, 2007.

[4] P. G. Esperante, C. Akamine, G. Bedicks, "Comparison of Terrestrial DTV Systems: ISDB-TB and DVB-T2 in $6 \mathrm{MHz}$," in IEEE Latin America Transactions, 2016, pp 45-56.

[5] R. Paderna, D. Q. Thang, Y. Hou, T. Higashino, M. Okada, "LowComplexity Compressed Sensing-Based Channel Estimation With Virtual Oversampling for Digital Terrestrial Television Broadcasting," in IEEE Transactions on Broadcasting, 2016, pp 82-91.

[6] Y. Iano, D. G. Caetano, R. Arthur, E. Carrara, C. Akamine, "Performance comparison of convolutional and turbo convolutional codes for ISDB-T in AWGN and Brazilian channels," presented at Telecommunications Symposium, Fortaleza, Ceará, Brazil, Sept. 3-6, 2006.

[7] Álvaro J. Almeida, Nuno A. Silva, Nelson J. Muga, Paulo S. André, Armando N. Pinto, "Calculation of the number of bits required for the estimation of the bit error ratio", in Proc. SPIE 9286, Portugal, 2014.

[8] M. Uehara, "Application of MPEG-2 Systems to Terrestrial ISDB (ISDB-T)," in Proc. of IEEE, 2006, pp 261-268.

[9] Longlong Wu, Yongjie Xie, Daping Xu, Li Ren, "Ballistic Missile Precession Frequency Extraction Based on the Viterbi \& Kalman Algorithm", in Proc. SPIE 9812, Enshi, China, 2015.
[10] [6] Oksana Volivach, Anatoly Beletsky, "Reed-Solomon's algorithm and software for correcting errors in a text", in Proc. SPIE 8008, Wilga, Poland, 2008.

[11] H. J. Santos, C. Sturm, J. Pontes. "Modulation and Detectation", in Radio Systems Engineering - A Tutorial Approach, $1^{\text {st }}$ ed. New York, USA.

[12] (2017) MATLAB software. [Online]. Available on: https://www.mathworks.com/products/matlab.html.

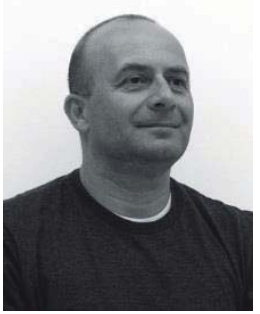

Adroaldo Raizer, was born in Lages, Santa Catarina, Brazil, on August 11, 1963. He received the titles of Electrical Engineer (1985) and master's degree in electrical engineering (1987), both from the Federal University of Santa Catarina. Completed his doctorate (1991) in Electrical Engineering by the Institut National Polytechnique de Grenoble, France. Currently Dr. Raizer is a full professor of the Department of Electrical and Electronic Engineering of the Federal University of Santa Catarina. He also works as coordinator of the Electromagnetic Compatibility and Engineering Group (GEMCO) and the Electromagnetic Compatibility and Electromagnetic Compatibility Laboratory (MagLab). His areas of interest are electromagnetic fields, electromagnetic compatibility and numerical methods.

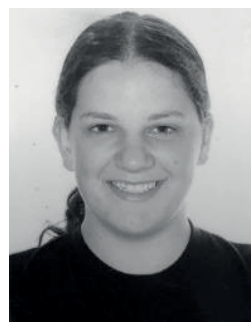

Kassia Toccolini, was born in Videira, Santa Catarina, Brazil, on April 18, 1991. She received the titles of Electronic Engineer (2016) by the Federal University of Santa Catarina. She is currently in master's degree in Electrical Engineering in electromagnetism area also in the Federal University of Santa Catarina. She is part of Electromagnetic Compatibility and Engineering Group (GEMCO) as a researcher. Her areas of interest are electromagnetic fields, telecommunication and digital signals processing.

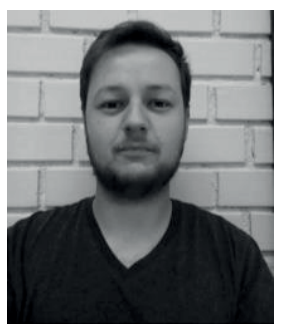

Mikael Pontes Fonseca, was born in Pelotas, Rio Grande do Sul, Brazil, on December 13, 1991. He received the title of Electronic Technician (2013) by the Federal Institute of Santa Catarina. He is currently Technical Manager of the Electromagnetism and Electromagnetic Compatibility Laboratory (MagLab). His areas of interest are electromagnetic fields, electromagnetic compatibility, automation, and embedded systems.

Received in 2017-06-30 | Approved in 2017-11-07 